\title{
CONF-970629..
}

\section{NANORHEOLOGY OF LIQUID ALKANES}

S. A. Gupta ${ }^{1,2}$, H. D. Cochran ${ }^{2,1}$, and P. T. Cummings ${ }^{1,2}$

${ }^{1}$ Department of Chemical Engineering

University of Tennessee

Knoxville, Tennessee 37996-2200

${ }^{2}$ Chemical Technology Division

Oak Ridge National Laboratory*

Oak Ridge, Tennessee 37831-6268

PECENER

OCT 081997

OSTI

Submitted to:

Fluid Phase Equilibria

September, 1997

The submiuted manuscript has been authored

by a contractor of the U.S. Govermment under contract No. DE-AC05-84OR22464.

Accordingly, the U.S. Govermment retains a nonexclusive, royalty-free license to publish or reproduce the published form of this contribution, or allow others to do so, for U.S. Government purposes.

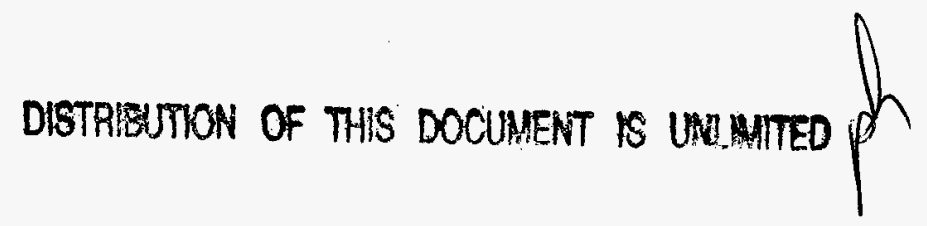

*Managed by Lockheed Martin Energy Rescarch Corp. for the U.S. Department of Energy under contract DE-AC05-960R22464. 


\section{DISCLAIMER}

This report was prepared as an account of work sponsored by an agency of the United States Government. Neither the United States Government nor any agency thereof, nor any of their employees, makes any warranty, express or implied, or assumes any legal liability or responsibility for the accuracy, completeness, or usefulness of any information, apparatus, product, or process disclosed, or represents that its use would not infringe privately owned rights. Reference herein to any specific commercial product, process, or service by trade name, trademark, manufacturer, or otherwise does not necessarily constitute or imply its endorsement, recommendation, or favoring by the United States Government or any agency thereof. The views and opinions of authors expressed herein do not necessarily state or reflect those of the United States Government or any agency thereof. 


\section{Nanorheology of Liquid Alkanes}

S. A. Gupta, H. D. Cochran, and P. T. Cummings*

Department of Chemical Engineering,

University of Tennessee,

Knoxville, TN 37996-2200

and

Chemical Technology Division, Oak Ridge National Laboratory,

Oak Ridge, TN 37831-6268

Submitted for publication in: Fluid Phase Equilibria

${ }^{*}$ Corresponding autlior. 


\section{Abstract}

We report molecular dynamics simulations of liquid alkanes, squalane and tetracosane, confined between moving walls to which butane chains are tethered, effectively screening the details of the wall. As in an experiment, heat is removed by thermostatting the tethered molecules. Results obtained at high strain rates, typical of practical applications, suggest little or no difference between the bulk rheology and confined flow, and the occurrence of a high degree of slip at the wall-fluid interface at the conditions studied. At relatively low velocities and high densities, tetracosane shows the formation of fully-extended chains at certain wall spacings.

Keywords: molecular simulation, rheology, molecular dynamics, alkanes

\section{Introduction}

Recent advances in experimental and computer simulation techniques for studying lubrication at the atomic scale have led to an increased understanding of fluids in ultra-thin films.

Surface force apparatus (SFA) experiments have clearly shown that the static and dynamic properties of fluids in molecularly thin films can be noticeably different from those of bulk fluids $[1,2]$. In general, bulk behavior persists until film thicknesses of order ten molecular diameters. Differences in the dynamic response from the bulk behavior are quite dramatic [1]. The effective viscosities of thin films rise orders of magnitude above the bulk values with a corresponding increase in the molecular relaxation times [3]. However, it should be recognized that dynamical experiments with extensions of the surface force apparatus are conducted at strain rates $\left(10-10^{5} \mathrm{~s}^{-1}\right)$, orders of magnitude below the strain rates which are found in the lubrication of disk drives, of micromachines, and of camshaft lifters in automobile engines $\left(c a .10^{8} \mathrm{~s}^{-1}\right)$ and which 
are only accessible computationally [4]. Table 1 compares the temperatures and strain rates for SFA experiments, prior bead-spring molecular dynamics (MD) simulations, and this study to those that exist in practical applications. The MD simulations of chains $[5,6,7,8]$ with the fully-flexible bead-spring model are performed at temperatures which are apparently very low. However, because of an apparent time-temperature scaling, the effective regime of the bead-spring system is similar to the experimental regime [4]. This scaling could explain the reasonable success of the bead-spring simulations in qualitatively reproducing many aspects of the experiments.

Some of the difficulties with realistic (as compared to bead-spring) simulations of confined fluid rheology involve realistic treatment of the specific wall-fluid interactions and of the thermostatting of the simulation. We have addressed these challenges with a novel simulation approach which we believe may also be amenable to experiment. We have studied the dynamic response of liquid alkanes confined between walls that have short alkane chains $\left(\mathrm{C}_{4} \mathrm{H}_{10}\right)$ tethered to them, which effectively screen the details of the wall. Shear flow is induced in the film by moving the walls, and heat is removed by thermostatting the first two atoms of the tethered chains. Our goal is to study the confined flow of alkanes of intermediate size range, comparable to many lubricants, and here we present some results obtained for tetracosane $\left(\mathrm{C}_{30} \mathrm{H}_{62}\right)$, which is a linear molecule, and squalane $\left(\mathrm{C}_{30} \mathrm{H}_{62}\right)$, a branched molecule with six symmetrically placed methyl side groups along a 24 carbon backbone. Detailed results from our simulations [9] will appear in a subsequent publication [10].

In Section 2 we present the molecular model and the simulation method, while Section 3 compares the rheological properties of the confined films to those of the bulk fluid, and presents some insights into the conformations of these molecules. 


\section{Molecular Model and Simulation Method}

We perform two types of simulations: (i) nonequilibrium molecular dynamics (NEMD) simulations in a confined geometry, and (ii) NEMD simulations for the bulk fluid. The bulk fluid simulations are performed at the same conditions as those that exist in the confined geometry, i.e. at the same temperature and strain rate. The density used for the bulk simulations is the average density in the central region of the confined geometry. Limited simulations also compare the confined and bulk results under constant pressure conditions.

\subsection{Potentials}

The model used for the alkanes is the united atom model previously used by Siepmann et al. $[11,12]$ and also by Mondello and Grest [13]. We have also used this model to successfully predict the Newtonian viscosities of various bulk fluid alkanes, including squalane [14]. The methyl and methylene groups are treated as spherical interaction sites with the interaction centers located at the centers of the carbon atoms. The interaction between atoms on different molecules and atoms separated by more than three bonds in the same molecule is described by the well-known Lennard-Jones (LJ) potential, with a cutoff distance of $2.5 \sigma_{\mathrm{CH}_{2}}$. The intramolecular interactions include a stretching term, a bending term and a torsional term. The bond stretching is described by a harmonic potential [15], with an equilibrium bond length of $1.54 \AA$ and a force constant $A / k_{B}=452,900 K / \AA^{2}$. The bond angle bending is also described by a harmonic potential while the torsional potential is that by Jorgensen et al. [16]. To prevent umbrella inversion of the $s p_{3}$ bond configuration at tertiary carbon branch points, we use a harmonic potential similar to the bending term, introduced by Mondello and Grest [13]. The LJ size and energy parameters, and the intramolecular interaction parameters can be found in [13]. 
For the atom-wall interactions, we use the potential proposed by Padilla and Toxvaerd [17], modified to model a structureless wall,

$$
U^{W}(y)=\frac{2 \pi \rho_{s} \epsilon_{w}}{3}\left(\frac{2 \sigma_{w}^{12}}{15 y^{9}}-\frac{\sigma_{w}^{6}}{y^{3}}\right)+10 \epsilon_{w} \exp \left[-21.0\left(\frac{y}{a_{1}}-y_{0}\right)\right]
$$

The parameters that characterize $U^{W}(y)$ are collected in Table 2. The size parameter $\sigma_{w}=0.5\left(\sigma_{s}+\sigma\right)$, where $\sigma_{s}=2.22 \AA$ determines the size of the "atoms" in the solid surface. The energy parameter is $\epsilon_{w}$, and $a_{1}$ is the lattice parameter. The exponential term in $y$ ensures the impenetrability of the wall. The interaction of every atom in the simulation with the wall is considered.

The first atom of each tethered chain is attached at a distance $w_{1}=0.673 \sigma_{\mathrm{CH}_{2}}$ from the wall by a harmonic potential with the same force constant as for the bond stretching term. We use a spacing of $2 a_{1}$ for the tethering sites on a square grid parallel to the walls. We choose to use a wall without structure because the tethered alkane chains effectively screen details of the wall.

\subsection{Simulation method}

For both the confined and bulk simulations, we use rRESPA multi-timestep dynamics $[18,19]$. For the bulk NEMD simulations, the motion of the particles can be described by the SLLOD algorithm [20]. The SLLOD equations of motion (incorporating a Nosé thermostat) for a system under planar Couette are given by

$$
\begin{aligned}
& \dot{\mathbf{r}}_{i a}=\frac{\mathbf{p}_{i a}}{m_{i a}}+\gamma y_{i a} \hat{x} \\
& \dot{\mathrm{p}}_{i a}=\mathbf{F}_{i a}-\gamma p_{y, i a} \hat{x}-\dot{\zeta} \mathbf{p}_{i a} \\
& \dot{\zeta}=\frac{p_{\zeta}}{Q} \\
& \dot{p}_{\zeta}=F_{\zeta}
\end{aligned}
$$

where $\mathbf{r}_{i a}$ and $\mathbf{p}_{i a}$ are the vector coordinates and peculiar momentum of atom $a$ in molecule $i, y_{i a}$ and $p_{y, i a}$ are its $y$ components, $\mathbf{F}_{i a}$, the force and $m_{i a}$, the mass. The strain rate of the imposed shear field is denoted by $\gamma, \hat{x}$ is a unit vector in the $x$ direction, $\zeta, p_{\zeta}$, and $Q$ are the variables related to the Nosé thermostat, $F_{\zeta}=\sum_{i, a} \frac{p_{i a}^{2}}{m_{i a}}-$ 
$3 N k_{B} T, Q=3 N k_{B} T \tau^{2}, \tau$ is the Nose thermostat time constant, and $N$ is the total number of atoms in the sistem.

For the confined simulations, the above equations apply, but without the $y$ terms for all atoms, and without thermostatting terms for all but the two atoms of each tethered molecule that are closest to the wall. The motion of the walls is simulated by moving the points of attachment of the tethered chains. The top and bottom walls move at velocities $\left(v_{\text {wall }}, 0,0\right)$ and $\left(-v_{\text {wall }}, 0,0\right)$, respectively.

\section{Results}

We present some results for the confined alkanes; magnitudes are presented in reduced units (length is reduced with $\sigma_{\mathrm{CH}_{2}}$, energy with $\epsilon_{\mathrm{CH}_{2}}$ and the mass with $m_{\mathrm{CH}_{2}}$ ) unless otherwise stated. The distance between the walls is $w=9.25$ and the number density $N / V=2.15$, where $V$ is defined as the dimensionless volume obtained by subtracting a volume $2 l^{2} w_{1}$ ( $l$ is the box-length along the wall) from the total volume between the walls, and $N$ is the total number of atoms in the simulation. The simulations at this wall spacing use 64 molecules of the alkane. We thermostat the first two atoms of the tethered $C_{4}$ molecules to $300 \mathrm{~K}$. Fig. 1 shows the velocity profile and the shear stress $\left\langle P_{y x}\right\rangle$ profile for the fluid in the confinement. Since the wall velocity is $v_{\text {wall }}=0.5$, Fig. 1 indicates considerable slip at the interface between the tethered chains and the confined fluid. Previous studies have reported observing a similar slip $[6,8]$. The shear stress $\left\langle P_{y x}\right\rangle$, which is calculated by the method of planes [21], is constant across the width, and agrees very well with the value computed as the average friction force between the fluid and the tethered chains per unit area of the walls, and also with the average heat dissipated by the thermostat per unit of time. We calculate the strain rate; $\gamma=\partial v_{x}(y) / \partial y$, by fitting the velocity profile to a straight line.

The data in Fig. 2 show the riscosities obtained for confined squalane and compares 
them with the bulk fluid values. Note the fluid undergoes shear thinning. The bulk viscosities are calculated at the same strain rates and temperatures as for the confined simulations (the temperature for the confined fluid was determined to be constant to within $2 \%$ across the gap). Remarkably, over the range of strain rates examined, the confined viscosity is identical to the bulk viscosity; the slight discrepancy seen at the lower strain rates has been determined to be due to differences between the calculated confined and bulk pressures; note the data point denoted by the solid triangle in Fig. 2, which is at the same pressure as the corresponding confined result. Stevens et al. [4] in their simulations of hexadecane report a similar observation, though the agreement between the bulk and confined viscosities is of a more qualitative nature. It must be pointed out that the results at various strain rates are all at different fluid temperatures (at the higher strain rates, there is significant viscous dissipation, which is negligible in SFA experiments). However, just as one would control the temperature in an experiment, in each case only the first two atoms of the tethered $C_{4}$ molecules are thermostatted at $300 \mathrm{~K}$.

Including the effect of the change in temperature with $\gamma$, the rate of shear thinning of the confined fluid follows an empirical power law, $\eta \propto \gamma^{-0.68}$, which is similar to results from experiments on shorter linear alkanes. The reported exponents are in the range -0.45 to $-0.7[3,22]$, albeit at lower strain rates, with onset of nonlinear response occurring between $10-100 \mathrm{~s}^{-1}$. We have performed additional simulations at high strain rates with a thermostat also for the confined fluid to remove heat generated by viscous dissipation and maintain a fluid temperature of $300 \mathrm{~K}$. A power-law fit to these 'constant temperature' data indicates a marginal effect on the exponent $\left(\eta \propto \gamma^{-0.60}\right)$. Fig. 2 also shows the viscosity of confined squalane for a smaller wall spacing $(=6.7)$ and at the same number density as for the wider spacing. The monomer density profiles indicate 3 and 5 layers respectively. For the two wall spacings, however, the strain rate 
dependent viscosities appear to coincide.

The results in Fig. 2 suggest that at high strain rates the rheological behavior of the confined fluid (regardless of wall spacing) is the same as that of the bulk fluid at the same temperature and strain rate. Concerning the structure of the molecules, our results indicate that the molecules are coiled up with the longest semi-axis being about $1.2 \mathrm{~nm}$. Reiter et al. [23] in their SFA experiments with squalane speculate that the molecules have a rather globular shape with a diameter of about $1.0 \mathrm{~nm}$. These results suggest that confined flow SFA experiments may yield a useful, perhaps even quantitative, model of practical lubrication applications because the SFA experiments impose a similar structure on the fluid yielding similar power law rheological behavior as in lubrication but at experimentally accessible strain rates. However, proper interpretation of the SFA experiments will require careful accounting for the slip at the wall.

Results for tetracosane also indicate that for the range of strain rates in our simulations, there is little or no difference between the bulk and confined viscosities. However, tetracosane shows a remarkably interesting behavior at high densities and at relatively low strain rates (when viscous dissipation is negligible). In spite of the absence of a crystalline solid surface, when the wall spacing is close to an integral multiple of the molecular diameter, the lack of side branches in tetracosane allows for the formation of very well-defined layers with the majority of the molecules being in a fully extended state. Fig. 3 shows the normalized density profile from a simulation of tetracosane at $v_{\text {wall }}=0.05$; the distance between the walls is $w=9.25$ and the number density $N / V \approx 2.15$ (the densities are normalized by the average density in the center). Note the five peaks of equal height. It is suggested that this tight packing of fully-extended chains is what gives rise to the high frictional forces that are observed in SFA experiments.

A future publication [10] will present further details of our simulations results. These 
results do not suggest either a crystallization or a glass transition of the confined alkanes. We compare the density profiles and chain conformations of squalane and tetracosane under similar conditions. We also examine how the molecular structure affects the interfacial slip behavior of these alkanes. In particular tetracosane shows a higher degree of slip than squalane, which is related to the conformational structure of the molecules. We present results which indicate that the shear thinning power-law exponent increases modestly with increasing density.

\section{Acknowledgments}

The authors gratefully acknowledge Gary Grest for suggesting the use of tethered chains; and Maurizio Mondello, Paz Padilla, and Shengting Cui for helpful discussions. The work of HDC and PTC has been supported in part by the Division of Chemical Sciences of the U. S. Department of Energy (DOE) at Oak Ridge National Laboratory (ORNL) and, by a grant to PTC, at the University of Tennessee. This work was sponsored as Laboratory Directed Research at ORNL. The authors acknowledge the use of the Intel Paragon supercomputers in the Center for Computational Sciences at ORNL, funded by

the DOE's Mathematical, Information, and Computational Sciences Division. ORNL is managed by Lockheed Martin Energy Research Corp. for the DOE under Contract No. DE-AC05-96OR22464.

\section{References}

[1] J. N. Israelachvili, P. M. McGuiggan, and A. M. Homola, Science, 240 (1988) 189-191.

[2] S. Granick and J. Van Alsten, Phys. Rev. Lett., 61 (1988) 2570-2573.

[3] H. Hu, G. A. Carson, and S. Granick, Phys. Rev. Lett., 66 (1991) 2758-2761. 
[4] M. J. Stevens, M. Mondello, G. S. Grest, S. T. Cui, H. D. Cochran, and P. T. Cummings, J. Chem. Phys., in press (1997).

[5] P. A. Thompson, G. S. Grest, and M. O. Robbins, Phys. Rev. Lett., 68 (1992) $3448-3451$.

[6] P. A. Thompson, M. O. Robbins, and G. S. Grest, Israel Journal of Chemistry, 35 (1995) 93-106.

[7] E. Manias, G. Hadziioannou, I. Bitsanis, and G. ten Brinke, Europhys. Lett., 24 (1993) 99-104.

[8] E. Manias, I. Bitsanis, G. Hadziioannou, and G. ten Brinke, Europhys. Lett., 33 (1996) 371-376.

[9] S. A. Gupta, Rheology of Confined Liquid Alkanes by Nonequilibrium Molecular Dynamics, Ph.D. Thesis, University of Tennessee, May 1997.

[10] S. A. Gupta, H. D. Cochran, and P. T. Cummings, in preparation (1997).

[11] J. I. Siepmann, S. Karaboni, and B. Smit, Nature, 365 (1993) 330-332.

[12] B. Smit, S. Karaboni, and J. I. Siepmann, J. Chem. Phys., 102 (1995) 2126-2140.

[13] M. Mondello and G. S. Grest, J. Chem. Phys., 103 (1995) 7156-7165.

[14] H. D. Cochran, P. T. Cummings, S. T. Cui, S. A. Gupta, R. K. Bhupathiraju and P. F. LoCascio, J. Comp. and Math. w. Appl., in press (1997).

[15] C. J. Mundy, J. I. Siepmann, and M. L. Klein, J. Chem. Phys., 102 (1995) 33763380.

[16] W. L. Jorgensen, J. D. Madura, and C. J. Swenson, J. Am. Chem. Soc., 106 (1984) 6638-6646. 
[17] P. Padilla and S. Toxvaerd, J. Chem. Phys., 101 (1994) 1490-1502.

[18] M. E. Tuckerman, B. J. Berne, and G. J. Martyna, J. Chem. Phys., 97 (1992) 1990-2001.

[19] S. T. Cui, P. T. Cummings, and H. D. Cochran, J. Chem. Phys., 104 (1996) 255262.

[20] D. J. Evans and G. P. Morris, Statistical Mechanics of Nonequilibrium Liquids, Academic, New York, 1990.

[21] B. D. Todd, D. J. Evans, and P. J. Daivis, Phys. Rev. E., 52 (1995) 1627-1638.

[22] G. Carson, H. Wu, and S. Granick, Tribology Trans., 35 (1992) 405-410.

[23] G. Reiter, A. L. Demirel, J. Peanasky, L. L. Cai, and S. Granick, J. Chem. Phys., 101 (1994) 2606-2615. 


\section{TABLES}

Table 1: Comparison of state points.

\begin{tabular}{|l|l|l|}
\hline & Temperature $\left({ }^{\circ} \mathrm{K}\right)$ & Strain rate $\left(\mathrm{s}^{-1}\right)$ \\
\hline Lubrication & $300-500$ & $\sim 10^{8}$ \\
SFA experiments & 300 & $10^{1}-10^{5}$ \\
Bead-spring MD & $\sim 100^{\dagger}$ & $10^{9}-10^{11}$ \\
This work & $300-500$ & $10^{8}-10^{11}$ \\
\hline
\end{tabular}

$\dagger$ Ref. [4]

Table 2: Atom-wall potential parameters.

$$
\begin{aligned}
& \sigma_{s}=2.22 \AA \\
& a_{1}=1.119 \sigma_{s} \\
& \rho_{s}=\sqrt{2} / a_{1}^{3} \\
& \sigma_{w}=0.5\left(\sigma_{s}+\sigma\right) \\
& \epsilon_{w} / k=80.0 \mathrm{~K} \\
& y_{0}=0.752242274
\end{aligned}
$$


Figure 1: Velocity (filled circles) and shear stress (open circles) profiles across the fluid film; wall spacing $=9.25, v_{\text {wall }}= \pm 0.5$. The dashed line is the linear fit to the velocity data, $\gamma=\partial v_{x}(y) / \partial y=0.0697$. The solid line is the average value of the shear stress calculated from the interaction of the fluid with the tethered chains.

Figure 2: Shear viscosity of confined and bulk squalane. The temperature of the fluid is indicated in each case. The straight line is a power-law fit to the data for the wider wall spacing, the exponent is -0.68 . The solid triangle depicts the calculated bulk viscosity at the same pressure as the corresponding confined result. For constant temperature results (not shown), the calculated exponent is -0.60 .

Figure 3: The normalized site density profile for tetracosane at a low velocity; $w=9.25$, $v_{\text {wall }}=0.05$. 


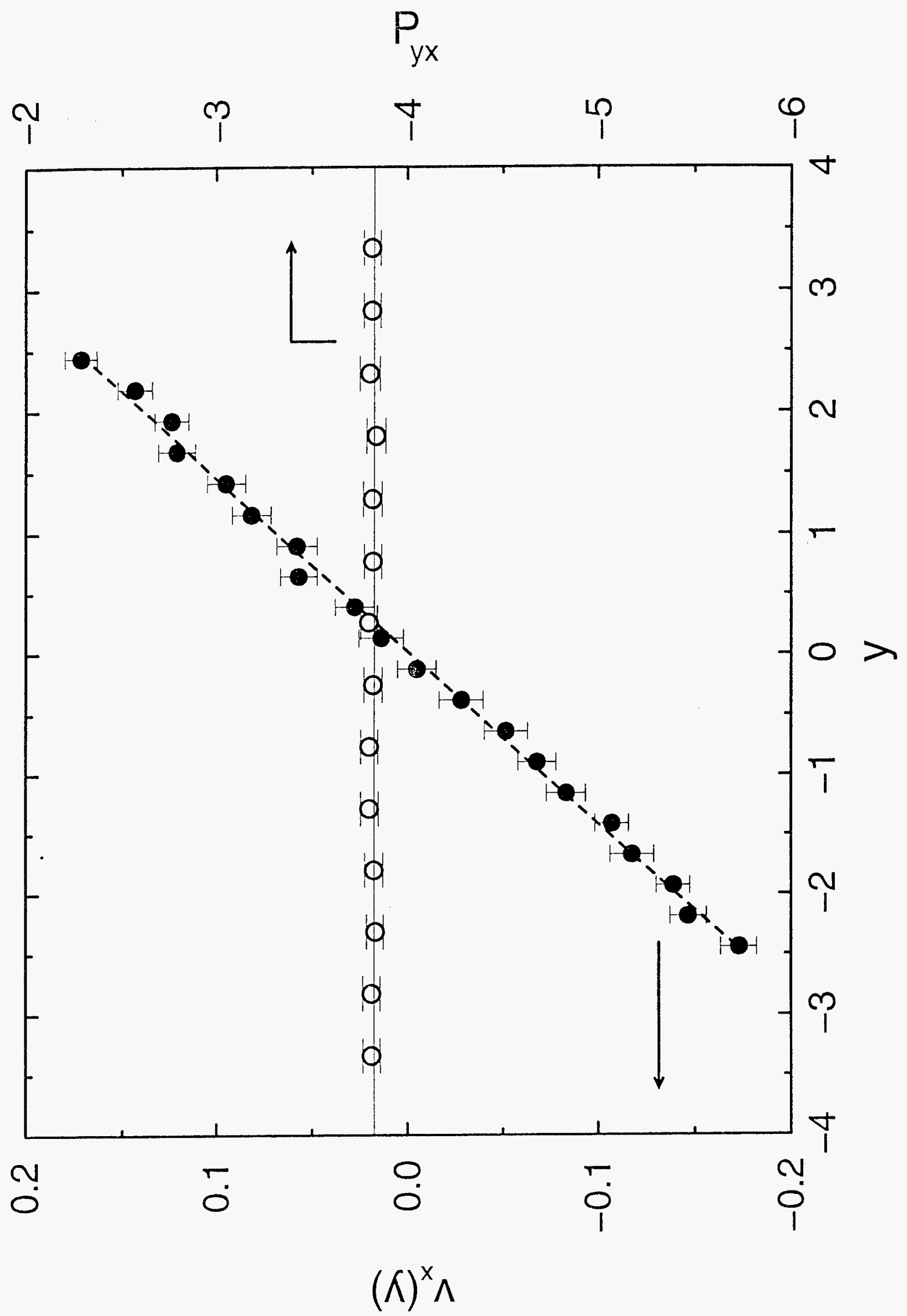




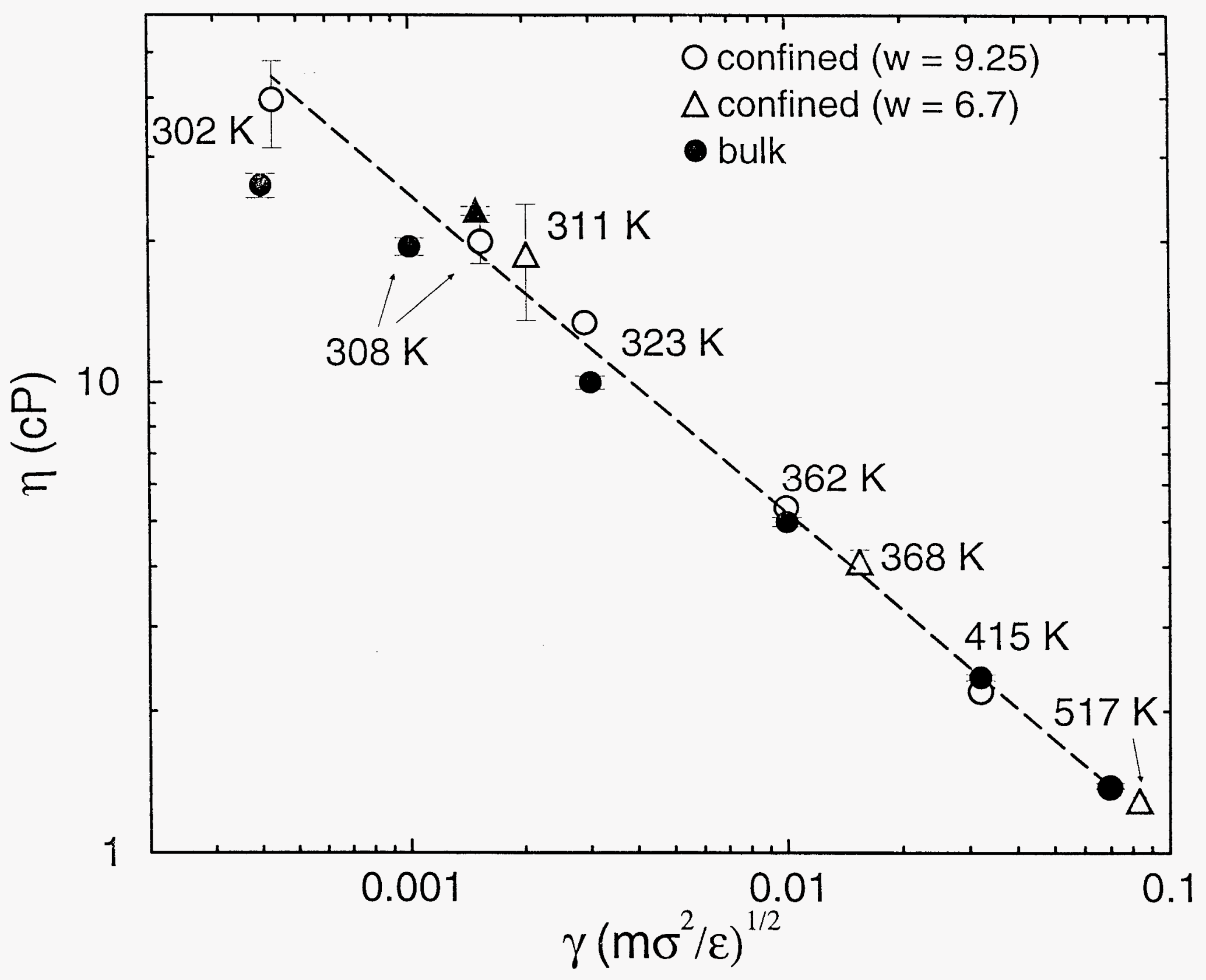




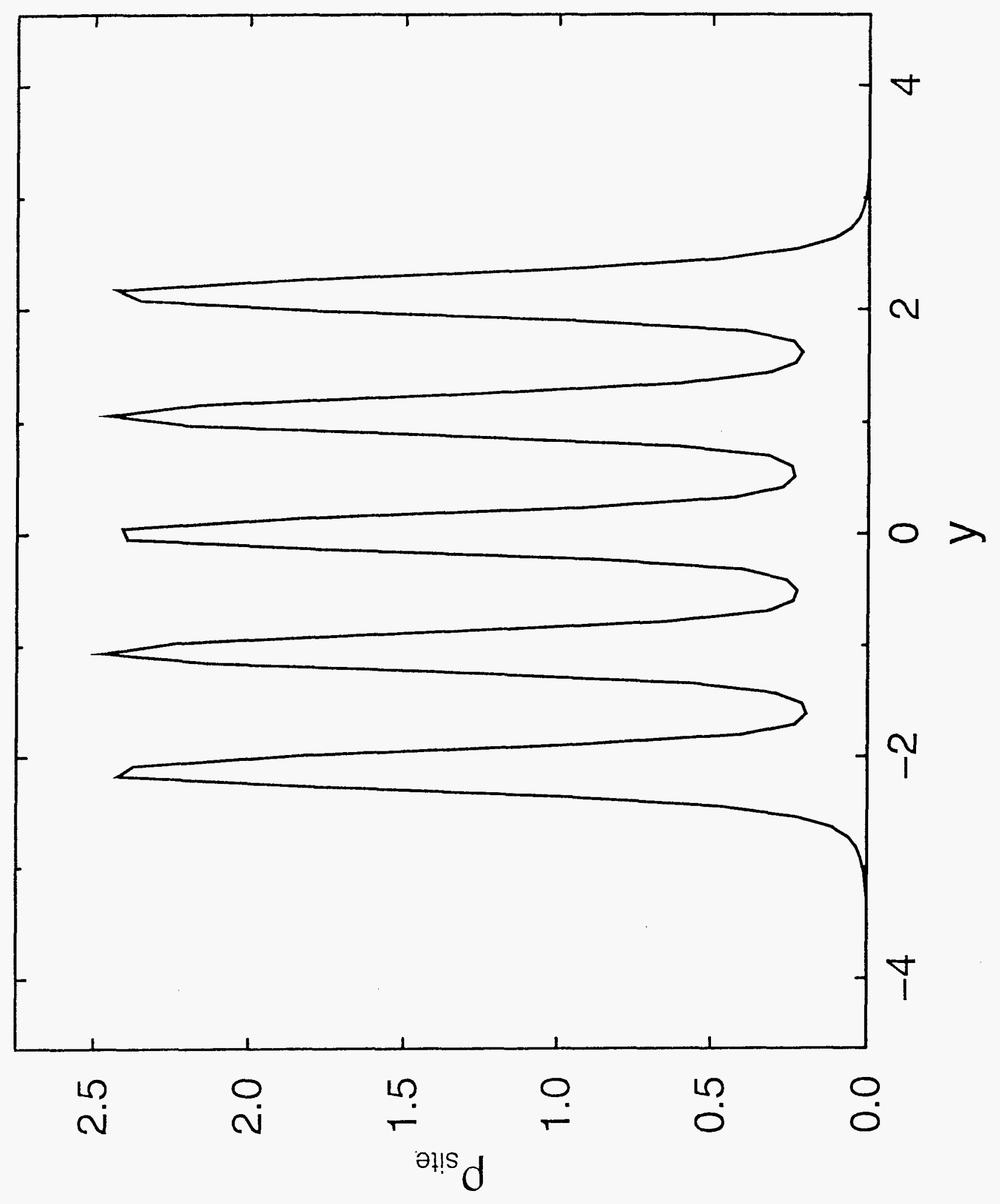


M9800036

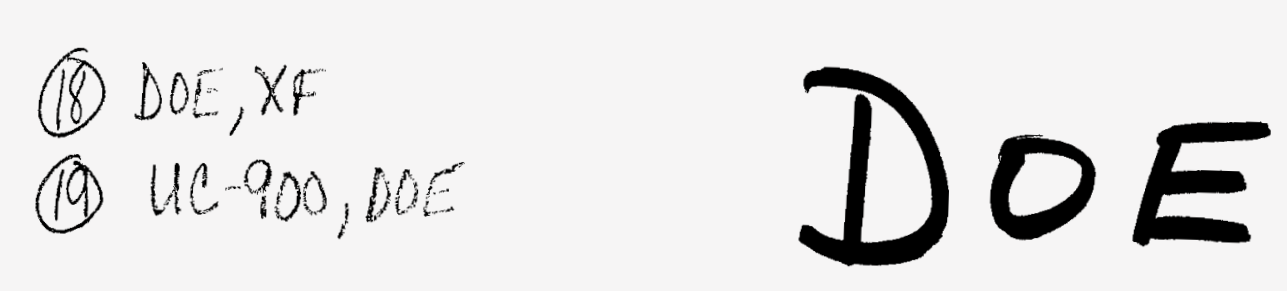

19971202068 